\title{
Episódios de Alta Frequência Atrial e sua Associação com Eventos Isquêmicos Cerebrais em Pacientes Chagásicos
}

\author{
Atrial High-Rate Episodes and Their Association with Cerebral Ischemic Events in Chagasic Patients
}

Emanoela Lima Freitas, ${ }^{1 \oplus}$ Elieusa e Silva Sampaio, ${ }^{2}{ }^{\circledR 0}$ Márcia Maria Carneiro Oliveira, ${ }^{2,3}{ }^{\circledR}$ Lucas Hollanda Oliveira, ${ }^{4}$ Marcos Sergio da Silva Guimarães, ${ }^{5}$ Jussara de Oliveira Pinheiro, ${ }^{5}$ Luís Pereira de Magalhães, ${ }^{5}$ Guisela Steffen Bonadie Albuquerque, ${ }^{1}$ Cristiano Macedo, ${ }^{50}$ Roque Aras $^{6(0)}$

Hospital Cardio Pulmonar, ${ }^{1}$ Salvador, BA - Brasil

Universidade Federal da Bahia - Enfermagem, ${ }^{2}$ Salvador, BA - Brasil

Escola de Enfermagem, ${ }^{3}$ Salvador, BA - Brasil

Hospital Ana Nery, ${ }^{4}$ Salvador, BA - Brasil

Hospital Universitário Professor Edgard Santos, ${ }^{5}$ Salvador, BA - Brasil

Universidade Federal da Bahia Faculdade de Medicina de Bahia, ${ }^{6}$ Salvador, BA - Brasil

\section{Resumo}

Fundamento: Episódios de alta frequência atrial (EAFAs) estão associados a um risco elevado de eventos isquêmicos cerebrais, porém não existem estudos relacionados com a presença de EAFAs e eventos isquêmicos cerebrais em pacientes chagásicos.

Objetivo: Investigar a associação entre a presença de EAFAs $\geq 6$ minutos e eventos isquêmicos cerebrais em pacientes chagásicos.

Métodos: Estudo de coorte com pacientes chagásicos, portadores de dispositivos cardíacos eletrônicos implantáveis (DCEls), acompanhados no ambulatório de arritmias de um hospital universitário, na cidade de Salvador/BA, entre maio de 2016 e junho de 2017. Pacientes com diagnóstico de flutter atrial/fibrilação atrial, com DCEI unicameral e em uso de anticoagulação oral foram excluídos. Foram considerados EAFAs com frequência atrial $\geq 190$ batimentos por minuto e duração $\geq 6$ minutos ( $\mathrm{min}$ ), e os eventos isquêmicos cerebrais foram identificados por meio de tomografia computadorizada (TC) de crânio.

Resultados: Os 67 participantes da pesquisa (67,2\% do sexo feminino, com idade média de 63,6 \pm 9,2 anos) foram acompanhados por $98 \pm 28,8$ dias e 11,9\% dos pacientes apresentaram EAFAs $\geq 6$ minutos. A TC de crânio evidenciou eventos isquêmicos cerebrais silenciosos em 16,4\% dos pacientes, sendo que, destes, $63,6 \%$ haviam apresentado os EAFAs $\geq 6$ minutos na análise dos DCEls. A idade avançada (OR 1,12 [IC 95\% 1,03-1,21; $p=0,009$ ) e a presença de EAFAs $\geq 6$ minutos (OR 96,2 [IC 95\% 9,4-987,4; $p<0,001]$ ) foram preditores independentes para eventos isquêmicos.

Conclusão: EAFAs detectados por DCEls estavam associados à presença de eventos isquêmicos cerebrais silenciosos em pacientes chagásicos. (Arq Bras Cardiol. 2020; 115(6):1072-1079)

Palavras-chave: Doença de Chagas/complicações; Flutter Atrial; Infarto Cerebral; Isquemia Encefálica; Marca-Passo Artificial; Tomografia Computadorizada/métodos.

\section{Abstract}

Background: Atrial high-rate episodes (AHREs) are associated with an increased risk of cerebral ischemic events; however, there are no studies related to the presence of AHREs and cerebral ischemic events in Chagasic patients.

Objective: To investigate the association between the presence of AHREs $\geq 6$ minutes and cerebral ischemic events in Chagasic patients.

Methods: Cohort study with Chagasic patients with implantable electronic cardiac devices (IECDs), followed at the Arrhythmia Outpatient Clinic of a University Hospital, in the city of Salvador, state of Bahia, Brazil, between May 2016 and June 2017.. Patients diagnosed with atrial flutter / atrial fibrillation, with unicameral IECD and using oral anticoagulation were excluded. AHREs with atrial frequency $\geq 190$ beats per minute and duration $\geq 6$ minutes (min) were considered, and cerebral ischemic events were identified by computed tomography (CT) of the skull.

Correspondência: Emanoela Lima Freitas •

Hospital Cardio Pulmonar - Unidade de Terapia Intensiva - Avenida Anita Garibaldi, 2199. CEP 40170-130, Ondina, Salvador, BA - Brasil E-mail: emanoela.limafreitas@gmail.com

Artigo recebido em 18/09/2019, revisado em 06/11/2019, aceito em 26/11/2019

DOI: https://doi.org/10.36660/abc.20190647 
Results: The 67 research participants (67.2\% females, mean age $63.6 \pm 9.2$ years) were followed for $98 \pm 28.8$ days and $11.9 \%$ of the patients had AHREs $\geq 6$ min. Skull CT showed silent cerebral ischemic events in 16.4\% of the patients, $63.6 \%$ of whom had AHREs $\geq 6$ min in the analysis of IECDs. Advanced age [OR 1.12 (95\% CI 1.03-1.21; p 0.009)] and the presence of AHREs $\geq 6$ minutes [OR 96.2 (95\% CI 9.4-987.4; p <0.001)] were independent predictors for ischemic events.

Conclusion: AHRES detected by IECDs were associated with the presence of silent cerebral ischemic events in Chagasic patients. (Arq Bras Cardiol. 2020; 115(6):1072-1079)

Keywords: Chagas Disease/complications; Atrial Flutter; Cerebral Infarction; Brain Ischemia; Pacemaker Artificial; Tomography, Computed/methods.

Full texts in English - http://www.arquivosonline.com.br

\section{Introdução}

A fibrilação atrial (FA) aumenta o risco de acidente vascular cerebral (AVC) isquêmico em cerca de 5 a 6 vezes, independentemente de outros fatores de risco. ${ }^{1}$ Nos últimos anos, tem crescido o interesse em detectar FA em uma fase mais precoce, antes da identificação clínica, com detecção principalmente por meio de marca-passo/ desfibrilador cardíaco implantável (CDI) e que precedem a primeira manifestação da doença. ${ }^{2,3}$ São os episódios de alta frequência atriais (EAFAs), que correspondem à ocorrência de arritmias atriais como fibrilação e flutter atrial, e são caracterizados por apresentar uma frequência atrial $\geq 190$ batimentos por minuto (bpm) ${ }^{4} \mathrm{ou} \geq 250 \mathrm{bpm},{ }^{3}$ com duração $\geq$ 5 a 6 minutos ( $\mathrm{min})$. São episódios assintomáticos e detectados apenas através de monitoramento contínuo, e denominados também "FA subclínica". ${ }^{4}$

EAFAs estão associados a um risco elevado de $\mathrm{AVC}^{5}$ e a tendência é que esses episódios tenham o mesmo prognóstico adverso que a FA clínica; no entanto, a duração, a frequência ou a carga diária exata desses episódios no risco de AVC ainda são desconhecidas; assim, o limiar de EAFAs que justifica a anticoagulação oral ainda não está claro. ${ }^{6}$

A incidência de EAFAs é de 30\% a $70 \%$, dependendo do perfil clínico da população estudada e dos algoritmos de detecção utilizados em cada protocolo de estudo. ${ }^{7}$ Ao excluir pacientes com história de FA e em uso de anticoagulação oral, esse número cai para cerca de $30 \% .^{8-10}$

Entretanto, em algumas populações específicas e vulneráveis às complicações tromboembólicas, como em pacientes portadores de doença de Chagas (DC), não existem dados relacionados à investigação da presença desses episódios e sua incidência.

Aproximadamente $30 \%$ dos pacientes com DC desenvolvem as alterações cardíacas, e mais de 10\%, alterações neurológicas. ${ }^{11}$ As complicações da doença cardíaca são principalmente em decorrência de arritmias e insuficiência cardíaca, responsáveis por mais de 35\% dos óbitos. ${ }^{12} \mathrm{Na}$ DC, a FA é a arritmia supraventricular mais frequente, sendo encontrada em 4 a $12 \%$ dos casos, ${ }^{13}$ sendo uma das principais causas dos eventos embólicos cerebrais, ${ }^{14}$ cuja incidência chega a atingir $3 \%$ da população chagásica. ${ }^{15}$

Investigar a presença dos EAFAs e sua associação com AVC em pacientes com DC pode permitir a inclusão desses pacientes na utilização de anticoagulantes orais. O objetivo foi investigar a associação entre a presença de EAFAs $\geq 6$ minutos e eventos isquêmicos cerebrais em pacientes chagásicos.

\section{Métodos}

\section{Desenho do Estudo e População}

Trata-se de um estudo observacional, do tipo coorte prospectiva. Foram incluídos 77 pacientes, de ambos os sexos, com idade $\geq 18$ anos, acompanhados no ambulatório de arritmias de um hospital universitário referência em Cardiologia, na cidade de Salvador/BA, entre maio de 2016 e junho de 2017. Os pacientes eram portadores de doença de Chagas e DCEls (marca-passo, cardiodesfibrilador implantável ou dispositivos de terapia de ressincronização cardíaca) capazes de monitorar a atividade atrial. Foram excluídos os pacientes com diagnóstico de fibrilação atrial/flutter atrial, portadores de DCEl unicameral, aqueles com indicação crônica de anticoagulação oral por qualquer razão ou que tinham contraindicação à realização de TC de crânio.

A pesquisa foi conduzida de acordo com os princípios da Declaração de Helsinque e aprovada pelo Comitê de Ética em Pesquisa do Hospital Universitário Professor Edgard Santos (UFBA-HUPES) (sob o número: 1.426.885, em 26/02/2016). O Termo de Consentimento foi obtido dos participantes.

Foram coletados dados sobre sexo, idade, etnia, comorbidades, tipo e indicação dos DCEls, modo de estimulação dos DCEls, caracterização da doença de Chagas, terapêutica medicamentosa, além de dados relacionados à radiografia de tórax, ecocardiograma transtorácico (ECO-TT) e eletrocardiograma (ECG) de longa duração - Holter 24 horas. A DC foi classificada, em cada paciente, conforme critérios do Consenso Brasileiro em Doença de Chagas.14 Os dados foram coletados a partir de entrevistas com os pacientes e prontuários.

Foi utilizado o escore de risco $\mathrm{CHA}_{2} \mathrm{DS}_{2}$-VASC - C: congestive heart failure or left ventricular systolic dysfunction (insuficiência cardíaca congestiva ou disfunção sistólica do ventrículo esquerdo) $=1$ ponto, $\mathrm{H}$ : hypertension (hipertensão) $=1$ ponto, $\mathrm{A}_{2}$ : age (idade) $\geq 75$ anos $=2$ pontos, $\mathrm{D}$ : diabetes mellitus (diabetes melito) $=1$ ponto, $\mathrm{S}_{2}$ : prior stroke or transient ischemic attack or thromboembolism (AVC prévio ou ataque isquêmico transitório ou tromboembolismo) $=2$ pontos, V: vascular disease (p. ex., peripheral artery disease, myocardial infarction, aortic plaque) (doença vascular [p. ex., doença arterial periférica, infarto do miocárdio, placa aórtica]) $=1$ ponto, A: age (idade) 65 a 74 anos $=1$ ponto e Sc: sex category (female sex) (gênero [sexo feminino]) $=1$ ponto. ${ }^{16}$

A classificação de risco de eventos cerebrovasculares, de acordo com o escore CHA2DS2-VASc é a seguinte: alto risco (2 pontos ou mais), risco intermediário (1 ponto) e baixo risco (0 ponto). 


\section{Procedimentos do Estudo}

Após assinatura do termo de consentimento informado, os pacientes foram submetidos à realização de ECG de 12 derivações, visando à confirmação da ausência de fibrilação atrial/flutter atrial, além da descrição do ritmo cardíaco e distúrbios de condução intraventricular. Em seguida, os pacientes foram avaliados por um médico arritmologista e tiveram seus DCEls ajustados para uma programação específica, visando à detecção e gravação das arritmias atriais.

Depois de um período de aproximadamente 3 meses após a programação, os pacientes retornaram ao ambulatório para análise dos dispositivos (leitura dos DCEIs), com o intuito de identificar e classificar a ocorrência das arritmias atriais, os EAFAs.

Durante o período entre a programação e a leitura dos DCEls, os pacientes foram submetidos à realização da TC de crânio sem contraste, com o objetivo de identificar eventos isquêmicos cerebrais. Foram identificados como infarto cerebral silencioso aqueles pacientes que apresentavam alterações de infarto cerebral nos laudos das TCs e que não tinham alterações clínicas de eventos isquêmicos ou déficits neurológicos. As TCs foram realizadas e avaliadas pelo departamento de neurorradiologia do hospital. Esse exame foi realizado em aparelho da Toshiba Medical Systems Corporation, 1385 (Shimo Ishigami, Otawara-Shi, Tochigi, Japão).

\section{Programação dos DCEls}

A escolha do fabricante do dispositivo não influenciou na inclusão/exclusão do paciente. Foram incluídos dispositivos dos fabricantes Medtronic $₫$, Stjude Medical ${ }^{\circledR}$ e Biotronik ${ }^{\circledR}$, cujos modelos estavam disponíveis para uso nessa população.

O dispositivo foi programado para identificar EAFAs com duração de pelo menos $190 \mathrm{bpm}$, com duração $\geq 6$ minutos, reconhecido como um ponto de corte apropriado para selecionar EAFAs e descartar a contração atrial prematura ou falsos eventos ${ }^{17}$ durante todo período de monitoramento. Essa duração foi escolhida por ser consistente com a metodologia de dois grandes estudos - Asymptomatic Atrial Fibrillation and Stroke Evaluation in Pacemaker Patients and the Atrial Fibrillation Reduction Atrial Pacing Trial (ASSERT) ${ }^{4}$ e The Relationship Between Daily Atrial Tachyarrhythmia Burden From Implantable Device Diagnostics and Stroke Risk $(\text { TRENDS })^{3}$ - que demonstraram a associação entre EAFAs, com duração de pelo menos 5 ou 6 minutos, e eventos isquêmicos cerebrais. $\mathrm{O}$ eletrograma de armazenamento foi ativado para confirmar a ocorrência dos EAFAs. Os pacientes tiveram a sensibilidade atrial programada para 0,1 a 0,5 milivolts $(\mathrm{mV})$.

Todos os EAFAs detectados pelo DCEI com duração $\geq 6$ minutos e com frequência $\geq 190 \mathrm{bpm}$ foram documentados e enviados para avaliação cega do especialista (médico eletrofisiologista). A ativação de outros desencadeantes do armazenamento de eletrogramas foi deixada a critério do médico, mas não foi apoiada pelo protocolo do estudo.

Além da programação dos dispositivos, foram coletados dados relacionados com parâmetros de estimulação/ detecção, percentuais de utilização atrial e ventricular, além de frequência cardíaca mínima e máxima.

\section{Acompanhamento}

Após aproximadamente 3 meses desde a programação do DCEI, o paciente retornou para a consulta subsequente e, nesse momento, foram coletados os dados relacionados com a detecção dos EAFAs.

Os eletrogramas atriais correspondentes aos EAFAs detectados foram avaliados por médico eletrofisiologista cego aos resultados das TCs de crânio. Tais eletrogramas foram categorizados como detecções adequadas ou inadequadas. As detecções inadequadas (ruídos, detecção da onda Farfield ventricular ou sincronização ventriculoauricular repetitiva e não reentrante) $)^{17,18}$ foram excluídas.

Foram definidas três categorias sem sobreposição da duração dos EAFAs: (1) sem EAFAs; (2) EAFAs com duração $<6$ minutos; (3) EAFAs $\geq 6$ minutos. A partir da detecção dos EAFAs com duração $\geq 6$ minutos, foram definidos 4 subgrupos: (1) EAFAs entre 6 minutos e 29 minutos; (2) EAFAs entre 30 minutos e 5 horas e 59 minutos; (3) EAFAs entre 6 horas e 23 horas e 59 minutos e (4) EAFAs $\geq 24$ horas. Esses cortes foram definidos de acordo com os valores preditivos positivos avaliados em uma análise do estudo ASSERT. ${ }^{19}$

Para EAFAs $\geq 6$ minutos e $\geq 190$ batimentos/min, o valor preditivo positivo foi de $82,7 \%$. O valor preditivo positivo aumentou para 93,2\%, 96,7\% e 98,2\% quando a duração do limiar foi prolongada para 30 minutos, 6 horas e 24 horas, respectivamente. $\mathrm{O}$ aumento da frequência cardíaca limiar para 250 batimentos/min diminuiu as detecções de falsopositivo, mas em menor medida, e adicionou-se apenas marginalmente ao valor preditivo positivo quando foram utilizadas longas durações de limiar. ${ }^{19}$

\section{Eventos Isquêmicos na Tomografia de Crânio}

Todas as TCs foram realizadas e avaliadas pelo departamento de neurorradiologia do hospital onde o estudo foi realizado, com o objetivo de identificar áreas compatíveis com eventos isquêmicos (áreas isquêmicas, infartos lacunares, glioses localizadas ou áreas hipodensas). Para pacientes sem história clínica ou déficits neurológicos anteriores, os eventos isquêmicos foram considerados como silenciosos. As TCs de crânio foram realizadas em aparelho da Toshiba Medical Systems Corporation, 1385, Shimoishigami, Otawara-Shi, Tochigu, Japão.

\section{Análise Estatística}

A análise estatística foi realizada utilizando o programa IBM SPSS, versão 21.0 (IBM, Armonk, New York). Os dados foram submetidos à análise estatística descritiva, por meio de medições de frequência (absolutas e relativas) para variáveis qualitativas. Para as variáveis quantitativas, utilizaram-se média e desvio-padrão ou mediana e intervalo interquartil, a depender da distribuição da variável, que foi testada por meio do teste de Shapiro-Wilk.

Para as variáveis categóricas, foi aplicado o teste $\chi^{2}$ ou teste exato de Fisher. $\mathrm{O}$ teste Student $\mathrm{t}$ não pareado foi utilizado para as médias das idades dos pacientes com EAFAs $\geq 6$ minutos e sem EAFAs $\geq 6$ minutos e para a comparação das 
médias da FEVE, e o teste de Mann-Whitney foi empregado para comparação do implante do DCEl em meses.

Os resultados foram apresentados por meio de odds ratio $(\mathrm{OR})$ e os seus respectivos intervalos de confiança de $95 \%$ (IC95\%). Um valor de $p<0,05$ foi considerado estatisticamente significante.

\section{Resultados}

\section{Características da População e Detecção dos Eafas}

Foram incluídos 77 pacientes na coorte - 10 foram excluídos, sendo que 7 apresentaram FA no momento da programação do dispositivo cardíaco, sendo iniciada anticoagulação oral (por definição do médico assistente do paciente); 2 foram a óbito antes da leitura do dispositivo e em 1 paciente foi identificado farfield ventricular durante leitura do DCEI. Ao final, 67 participantes cumpriram todas as etapas da pesquisa.

A média de idade foi de 63,6 \pm 9,2 anos; todos os participantes estavam na fase crônica da DC, sendo que $89,6 \%$ deles haviam desenvolvido a forma cardíaca e $10,4 \%$ deles desenvolveram a forma cardiodigestiva da doença. Dentre as manifestações clínicas da forma cardíaca da DC, estavam presentes: síndrome arrítmica (100\% dos pacientes), insuficiência cardíaca (IC) (38,8\%) e síndrome tromboembólica (3\%, correspondendo a 2 pacientes com AVC prévio). As manifestações clínicas relacionadas com a forma cardiodigestiva consistiram em ocorrência de megaesôfago chagásico em 7 pacientes.

Quanto aos dados relacionados com DCEls, identificamos que $46,3 \%$ dos pacientes estavam em modo de estimulação DDD (estimulação de dupla câmara), seguido de 41,8\% do modo DDD-R (DDD com resposta de frequência - R); a frequência cardíaca mínima foi de 61,8 \pm 3,9 bpm, e máxima de 124,4 $\pm 5,5 \mathrm{bpm}$. O percentual de utilização atrial teve média de 52,8 $\pm 37,4 \%$ e o ventricular, $65,6 \pm 42,5 \%$.

A média de acompanhamento foi de $98 \pm 28,8$ dias e foram detectados EAFAs em 24 (35,8\%) pacientes, com durações variadas. A incidência de EAFAs com duração $\geq 6$ minutos ou "FA subclínica" foi de 11,9\% $(\mathrm{n}=08)$.

A mediana de tempo para atingir o primeiro EAFA foi de 26,2 dias (variando entre 0,08 e 83,25 dias), e a mediana da duração dos EAFAs foi de 135,4 minutos (variando entre 22,8 e 5.811,8 minutos).

As comparações das características demográficas e clínicas dos pacientes com EAFAs $\geq 6$ minutos versus pacientes sem EAFAs ou com duração $<6$ minutos são demonstradas na Tabela 1.

\section{Detecção dos Eventos Isquêmicos}

Onze pacientes (16,4\%) apresentaram evento isquêmico na TC de crânio e não tinham história de AVC prévio. Observouse que $87,5 \%$ dos pacientes que tinham EAFAs $\geq 6$ minutos, apresentaram eventos isquêmicos na TC de crânio. Na Tabela 2, demonstram-se as características clínicas dos pacientes com e sem eventos isquêmicos.
Identificou-se que $45,5 \%$ dos pacientes com eventos isquêmicos apresentaram EAFAs com duração entre 30 minutos e 5 horas e 59 minutos, e o número médio de EAFAs $\geq 6$ minutos foi de $3,88 \pm 2,58$, sendo que $50 \%$ dos pacientes tiveram entre 1 e 3 episódios.

Além de considerar o maior EAFA identificado pelo DCEI, foi mensurada também a carga diária total de EAFAs (o máximo de tempo em que o paciente permaneceu em "FA subclínica" em 24 horas) e sua possível associação com eventos isquêmicos. Evidenciou-se que o paciente chagásico com carga diária com duração $\geq 6$ minutos tem mais chance de desenvolver eventos isquêmicos (OR: 46,67 [6,57 - 331,67; p <0,001]). A mediana da carga diária máxima que teve associação com a ocorrência de eventos isquêmicos foi de 4.554 segundos (75,9 minutos) (OR: 1,001; $p<0,026)$.

A Tabela 3, mostra a descrição da carga diária de EAFAs em pacientes com e sem eventos isquêmicos.

A idade avançada e a presença de EAFAs $\geq 6$ minutos foram associadas à eventos isquêmicos, conforme demonstrado na Tabela 4.

\section{Discussão}

Houve uma associação de EAFAs $\geq 6$ minutos com eventos isquêmicos silenciosos. O estudo de Benezet-Mazuecos et al. ${ }^{20}$ evidenciou que eventos isquêmicos cerebrais silenciosos ocorrem mais nos pacientes com EAFAs (42\%) do que naqueles sem EAFAs (19\%), encontrando uma OR de 3,4. BenezetMazuecos et al. ${ }^{21}$ também descreveram que os eventos isquêmicos cerebrais silenciosos ocorreram mais nos pacientes com EAFAs (32\%) do naqueles sem EAFAs (13\%), encontrando uma OR de 2,45. Além disso, breves episódios de FA subclínica (48 horas) documentados pelo monitoramento do Holter foram associados a um risco significativamente aumentado de eventos isquêmicos cerebrais silenciosos e AVC. ${ }^{22}$

A incidência de EAFAs em pacientes sem história de FA é de cerca de $30 \%$, em períodos de monitoramento variados. ${ }^{4,9,10}$ Em nosso estudo, a incidência encontrada na população chagásica (também sem história de FA), em um período de monitoramento de cerca de 3 meses, foi de 11,9\%. Tal achado é muito similar ao encontrado em um grande estudo de 2.580 pacientes não chagásicos e sem história de FA, em que os EAFAs $\geq 6$ minutos foram encontrados em 35\% dos pacientes em um seguimento médio de 2,5 anos, e em 10\% dos pacientes nos primeiros 3 meses do estudo. ${ }^{4}$ No entanto, é importante ressaltar que a mediana de tempo para detecção dos episódios no nosso estudo com pacientes chagásicos (26,2 dias) foi inferior a de outros estudos, como no MOde Selection Trial (MOST) - 100 dias $^{2}$ - e no ASSERT - 36 dias. $^{4}$ Além disso, o número médio de EAFAs no nosso estudo foi maior $(3,9)$ que o do estudo ASSERT $(2,0){ }^{4}$

Neste estudo, todos os pacientes possuíam um DCEI dupla câmara; entretanto, não se encontrou associação entre o modo de estimulação dupla câmara e a presença ou ausência de EAFAs, e isso pode ser devido ao fato de que muitos episódios foram de curta duração. No entanto, é possível que em uma amostra maior de pacientes, uma redução dos EAFAs, com estimulação da dupla câmara possa ser detectada. O estudo MOST² também não evidenciou essa associação. 


\section{Artigo Original}

Tabela 1 - Características demográficas e clínicas dos pacientes com EAFAs $\geq 6$ minutos versus pacientes sem EAFAs ou com duração $<6$ minutos

\begin{tabular}{|c|c|c|c|c|}
\hline Características demográficas e clínicas & $\begin{array}{l}\text { População total } \\
\qquad(n=67)\end{array}$ & $\begin{array}{c}\text { Com EAFAs } \geq 6 \text { minutos } \\
\qquad(n=08)\end{array}$ & $\begin{array}{l}\text { Sem EAFAs ou }<6 \mathrm{~min} \\
(n=59)\end{array}$ & Valor de $p$ \\
\hline Idade (anos) - média \pm DP & $63,6 \pm 9,2$ & $69,9 \pm 10,4$ & $62,8 \pm 8,8$ & $0,040^{\dagger}$ \\
\hline Sexo $-n(\%)$ & & & & 0,103 \\
\hline Feminino & $45(67,2)$ & $3(6,7)$ & $42(93,3)$ & \\
\hline Masculino & $22(32,8)$ & $5(22,7)$ & $17(77,3)$ & \\
\hline Etnia - n (\%) & & & & 1,000 \\
\hline Branco & $4(6)$ & $0(0,0)$ & $4(100)$ & \\
\hline Não branco & $63(94)$ & $8(12,7)$ & $55(87,3)$ & \\
\hline Tipo de DCEI & & & & 1,000 \\
\hline Marca-passo & $62(92,5)$ & $8(12,9)$ & $54(87,1)$ & \\
\hline $\mathrm{CDI}$ & $5(7,5)$ & $0(0,0)$ & $5(100)$ & \\
\hline Indicação do DCEI - n (\%) & & & & 1,000 \\
\hline BAV/BAVT & $52(77,6)$ & $7(13,5)$ & $45(86,5)$ & \\
\hline DNS & $10(14,9)$ & $1(10,0)$ & $9(90)$ & \\
\hline Prevenção secundária MSC & $5(7,5)$ & $0(0,0)$ & $5(100)$ & \\
\hline Implante do DCEI (meses) $)^{\S}$ & $108(48-168)$ & $144(54-165)$ & $96(36-180)$ & $0,757^{\star}$ \\
\hline IC (NYHA) & $26(38,8)$ & $4(15,4)$ & $22(84,6)$ & 0,701 \\
\hline Classe funcional da IC & & & & 1,000 \\
\hline I & $7(26,9)$ & $1(14,3)$ & $6(85,7)$ & \\
\hline$\|$ & $13(50)$ & $2(15,4)$ & $11(84,5)$ & \\
\hline III & $6(23,1)$ & $1(16,7)$ & $5(83,3)$ & \\
\hline $\mathrm{IAM}-\mathrm{n}(\%)$ & $4(6)$ & $8(12,7)$ & $4(100)$ & 1,000 \\
\hline HAS - n (\%) & $50(74,6)$ & $6(12)$ & $44(88)$ & 1,000 \\
\hline Diabetes - n (\%) & $6(9)$ & $1(16,7)$ & $5(83,3)$ & 0,549 \\
\hline Dislipidemia - n (\%) & $21(31,3)$ & $2(9,5)$ & $19(90,5)$ & 1,000 \\
\hline FEVE $\%$ - média \pm DP & $58,5 \pm 14,1$ & $58,1 \pm 11$ & $58,6 \pm 14,5$ & 0,495 \\
\hline$A E \geq 40 m m-n(\%)$ & $22(32,8)$ & $3(13,6)$ & $19(86,4)$ & 1,000 \\
\hline Escore CHA2DS2-VASc & & & & 0,346 \\
\hline Risco baixo & $12(17,9)$ & $1(8,3)$ & $11(91,7)$ & \\
\hline Risco intermediário & $37(55,2)$ & $3(8,1)$ & $34(91,9)$ & \\
\hline Risco alto & $18(26,9)$ & $4(22,2)$ & $14(77,9)$ & \\
\hline
\end{tabular}

Fonte: próprio autor

Os dados são apresentados em média \pm desvio-padrão ou o número de pacientes (\%). §Dados apresentados em mediana e intervalo interquartil. Os valores de $P$ foram calculados usando o teste qui-quadrado. *Mann-Whitney e o tteste $t$ de Student, conforme apropriado.

DCEl: dispositivo cardiaco eletrônico implantável; CDI: cardiodesfibrilador implantável; BAV/BAVT: bloqueio atrioventricular/bloqueio atrioventricular total; DNS: doença do nó sinusal; MSC: morte súbita cardiaca; IC: insuficiência cardíaca (NYHA: New York Heart Association); IAM: infarto agudo do miocárdio; HAS: hipertensão arterial sistêmica; FEVE: fração de ejeção do ventrículo esquerdo; AE: átrio esquerdo; CHA2DS2-VASc: escore de risco de eventos tromboembólicos (C: congestive heart failure or left ventricular systolic dysfunction (insuficiência cardíaca congestiva ou disfunção sistólica do ventrículo esquerdo) $=1$ ponto, $\mathrm{H}$ : hypertension (hipertensão) $=1$ ponto, $A_{2}$ : age (idade) $\geq 75$ anos $=2$ pontos, $D$ : diabetes mellitus (diabetes melito) $=1$ ponto, $S_{2}$ : prior stroke or transient ischemic attack or thromboembolism (AVC prévio ou ataque isquêmico transitório ou tromboembolismo) $=2$ pontos, V: vascular disease (p. ex., peripheral artery disease, myocardial infarction, aortic plaque) (doença vascular [p. ex., doença arterial periférica, infarto do miocárdio, placa aórtica]) = 1 ponto, $A$ : age (idade) 65 a 74 anos = 1 ponto e Sc: sex category (female sex) (gênero [sexo feminino]) = 1 ponto). 
Artigo Original

Tabela 2 - Características clínicas dos pacientes com e sem eventos isquêmicos

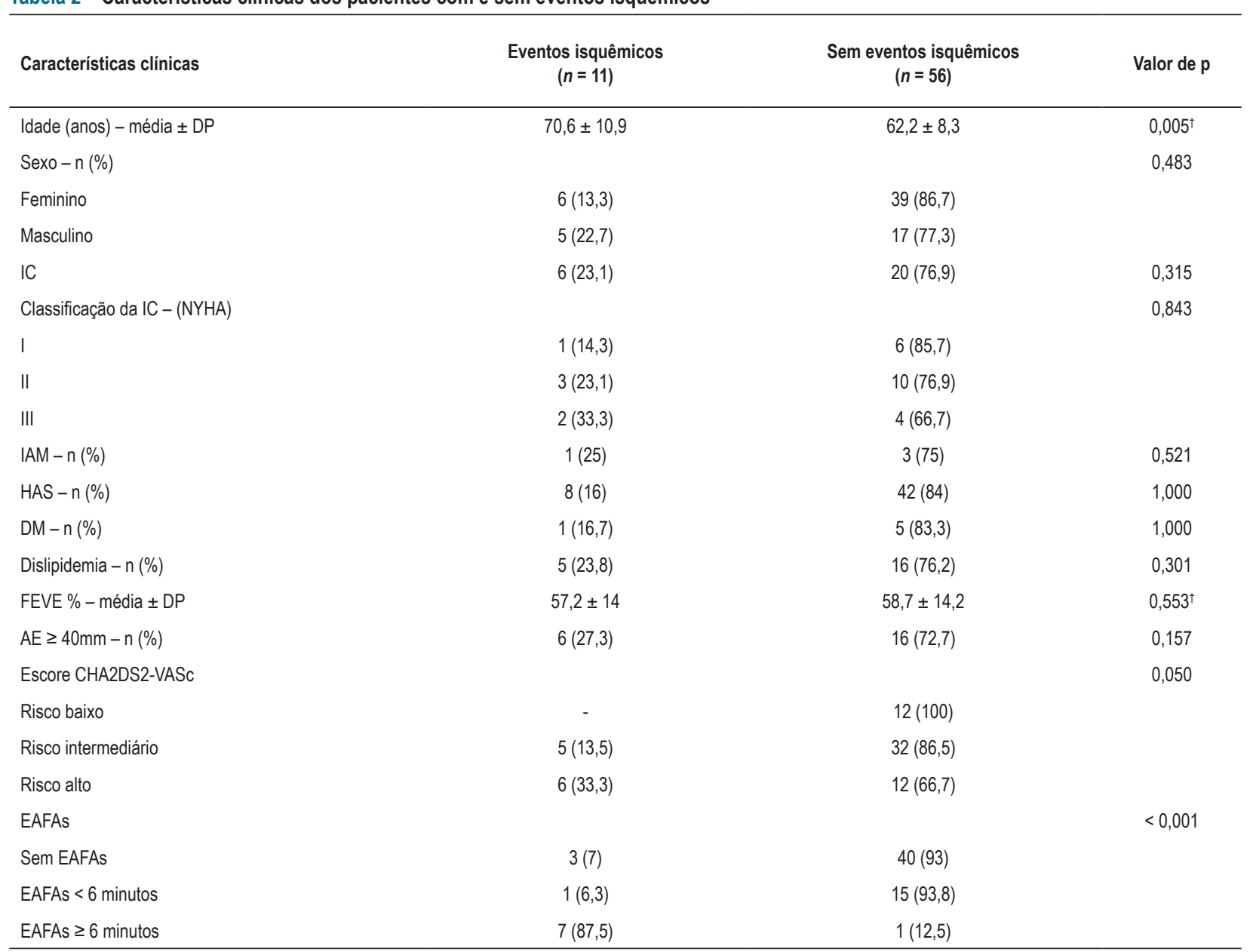

Fonte: próprio autor

Os dados são apresentados em média \pm desvio-padrão ou o número de pacientes (\%). §Dados apresentados em mediana e intervalo interquartil. Os valores de $P$ foram calculados usando o teste do qui-quadrado e o tteste $t$ de Student. (§ - não consta esse símbolo na tabela)

EAFAs: episódios de alta frequência atrial; DCEl: dispositivo cardíaco eletrônico implantável; MP: marca-passo cardiaco; BAV/BAVT: bloqueio atrioventricular/ bloqueio atrioventricular total; DNS: doença do nó sinusal; MSC: morte súbita cardíaca; IC: insuficiência cardíaca; NYHA: New York Heart Association; IAM: infarto agudo do miocárdio; HAS: hipertensão arterial sistêmica; DM: diabetes melito; FEVE: fração de ejeção do ventrículo esquerdo; AE: átrio esquerdo; CHA2DS2-VASc: escore de risco de eventos tromboembólicos (C: congestive heart failure or left ventricular systolic dysfunction (insuficiência cardiaca congestiva ou disfunção sistólica do ventrículo esquerdo) $=1$ ponto, $\mathrm{H}$ : hypertension (hipertensão) $=1$ ponto, $A_{2}$ : age (idade) $\geq 75$ anos $=2$ pontos, $D$ : diabetes mellitus (diabetes melito) $=$ 1 ponto, $S_{2}$ : prior stroke or transient ischemic attack or thromboembolism (AVC prévio ou ataque isquêmico transitório ou tromboembolismo) 2 pontos, V: vascular disease (p. ex., peripheral artery disease, myocardial infarction, aortic plaque) (doença vascular [p. ex., doença arterial periférica, infarto do miocárdio, placa aórtica]) $=1$ ponto, A: age (idade) 65 a 74 anos = 1 ponto e Sc: sex category (female sex) (gênero [sexo feminino]) = 1 ponto).

Tabela 3 - Descrição da carga diária de EAFAs em pacientes com e sem eventos isquêmicos

\begin{tabular}{lccc}
\hline Descrição da carga diária & $\begin{array}{c}\text { Com eventos } \\
\text { isquêmicos } \\
(n=11)\end{array}$ & $\begin{array}{c}\text { Sem eventos } \\
\text { isquêmicos } \\
(n=56)\end{array}$ & $\begin{array}{c}\text { Valor } \\
\text { de p }\end{array}$ \\
\hline Carga diária de EAFAs $-\mathrm{n}(\%)$ & $3(27,3)$ & $40(71,4)$ & $<0,001$ \\
Sem carga diária & $1(9,1)$ & $14(25)$ & \\
$<6$ minutos & $7(63,6)$ & $2(3,6)$ & \\
$\geq 6$ minutos & & & \\
\hline
\end{tabular}

Os dados são apresentados através do número de pacientes (\%). O valor de $\mathrm{p}$ foi calculado por meio do teste exato de Fischer. EAFAs: episódios de alta frequência atrial.
Tabela 4 - Fatores associados aos eventos isquêmicos

\begin{tabular}{llll}
\hline Fatores associados & OR & IC $95 \%$ & Valor de $\mathbf{p}$ \\
\hline EAFAs $\geq 6$ minutos & 96,2 & $9,4-987,5$ & $<0,001$ \\
Idade avançada & 1,12 & $1,03-1,21$ & 0,009 \\
IC & 2,16 & $0,58-7,98$ & 0,241 \\
Dislipidemia & 2,08 & $0,56-7,80$ & 0,270 \\
HAS & 0,89 & $0,21-3,82$ & 0,874 \\
\hline
\end{tabular}

OR: odds ratio; IC95\%: intervalo de confiança de 95\%; Teste $\chi^{2}$; EAFAs: episódios de alta frequência atrial; IC: insuficiência cardíaca; HAS: hipertensão arterial sistêmica. 
A detecção dos EAFAs foi maior em pacientes com idade avançada, do sexo feminino, de etnia negra, com passado de IAM, cuja indicação do implante do DCEI foi o BAV/ BAVT; contudo, apenas a idade avançada foi associada ao evento isquêmico. O envelhecimento da população traz consigo o aumento da doença cardíaca subjacente, e a melhora das técnicas de diagnóstico tem evidenciado uma alta prevalência de FA em pessoas com mais de 70 anos de idade. ${ }^{23}$ Isso também acontece com a população chagásica, ${ }^{24}$ onde a prevalência de FA foi acentuadamente maior com a idade avançada. Estudos evidenciaram que EAFAs eram mais prevalentes na população idosa, mas não foi encontrado significância estatística nessa associação. ${ }^{4,20}$

Além dos eventos isquêmicos silenciosos associados com EAFAs $\geq 6$ minutos em pacientes mais velhos, também houve associação do escore CHA2DS2-VASC de risco elevado com eventos isquêmicos silenciosos, semelhantes a outros estudos..$^{20,21,25}$

Apesar de os EAFAs detectados por DCEls estarem associados a um aumento de 2 a 2,5 vezes no risco de AVC, se comparados com indivíduos sem EAFAs, ${ }^{3,4}$ o risco absoluto de AVC entre esses pacientes é menor do que entre os portadores de FA clínica detectada. ${ }^{26}$

Os estudos de detecção da "FA subclínica" através de DCEls têm tentado identificar o limite de tempo ideal (considerando o maior episódio ou a carga diária) e suas consequências clínicas, tais como eventos tromboembólicos. As durações dos limiares descritos nos estudos têm sido altamente variáveis: 5 minutos, ${ }^{2,3}$ sugerindo aumento de 2,8 no risco de AVC ou óbito; 6 minutos, ${ }^{4}$ com aumento de 2,5 maior risco para tromboembolismo; 24 horas, ${ }^{8}$ com aumento de 3,1 com maior risco para eventos tromboembólicos. Da mesma forma, a carga diária de $3,8^{27}$ e 5,5 horas $^{3}$ também tem sido associada a um aumento significativo do risco de AVC (aumento de 9 e 2 vezes, respectivamente).

No nosso estudo, a carga diária dos EAFAs com duração $\geq 6$ minutos sugere maior chance de o paciente chagásico desenvolver evento isquêmico silencioso, um risco mais elevado do que o descrito por outros estudos. Em pacientes com DCEls, ${ }^{28}$ evidenciou-se uma OR de 2,11 para a ocorrência de eventos isquêmicos nos pacientes que tiveram pelo menos 1 dia com no mínimo 1 hora da carga de "FA subclínica" (IC 95\%: 1,22-3,64, p $=0,008$ ). Outro estudo ${ }^{20}$ descreve que a carga detectada pelos DCEls demonstrou-se significativamente associada a eventos cerebrais isquêmicos silenciosos, com OR 5,38.

A duração do maior episódio ou a carga diária dos EAFAs que elevam o risco de eventos isquêmicos suficientemente para justificar a anticoagulação são incertas. A recomendação atual é seguir o algoritmo de gerenciamento dos EAFAs7, visto que não existem estudos publicados até o momento sobre esse assunto.

\section{Limitações}

O estudo apresentado tem limitações relacionadas ao pequeno número de pacientes incluídos e ao seu desenvolvimento em um centro único de observação, mas vale ressaltar que não existe qualquer estudo sendo realizado com a população chagásica e com este objetivo. Além disso, diferentemente de outros estudos já realizados, foram incluídos aqui as três marcas de DCEls mais utilizadas pela população estudada, não se limitando a um único tipo de dispositivo. A avaliação destes (programação realizada e análise dos registros) não interferiu em demais configurações, atendendo, portanto, às necessidades dos pacientes durante todo o tempo de monitoramento, e sendo o estudo facilmente reprodutível.

Ausência de um grupo controle também corresponde a uma limitação; contudo, mesmo sem o grupo controle, vale frisar a contribuição relevante do estudo para obtenção de informações inéditas sobre a doença de Chagas e os eventos isquêmicos.

A investigação dos eventos isquêmicos foi realizada por meio de TC de crânio. A sensibilidade para a detecção da isquemia é maior a partir de ressonância magnética (RM) de crânio. Entretanto, a utilização de RM específica para pacientes em uso de DCEls ainda não é uma realidade para pacientes atendidos pelo sistema de saúde público brasileiro, e não foram realizadas análises de variabilidade intra e interobservador entre os médicos neurologistas.

\section{Conclusão}

Observamos que em pacientes com doença de Chagas e DCEls, os EAFAs $\geq 6$ minutos são frequentes e sua associação com eventos isquêmicos silenciosos foi significante. A ocorrência de eventos isquêmicos silenciosos também se associou a uma maior carga diária máxima. Essa associação foi mais prevalente em pacientes idosos, e as demais características da doença de Chagas não interferiram nos resultados avaliados.

Estes são os primeiros resultados em pacientes chagásicos já publicados, e podem oferecer subsídios para profissionais que realizam rotineiramente o acompanhamento desses pacientes, tornando-os conscientes da relevância desses episódios e direcionando-os na busca e na aplicação de algoritmos para essa população específica.

\section{Contribuição dos Autores}

Concepção e desenho da pesquisa: Freitas EL, Sampaio ES, Oliveira MMC, Aras R; Obtenção de dados: Freitas EL, Oliveira LH, Guimarães MSS, Pinheiro JO, Magalhães LP, Albuquerque GSB, Aras R; Análise e interpretação dos dados: Freitas EL, Oliveira MMC, Oliveira LH, Guimarães MSS, Pinheiro JO, Magalhães LP, Albuquerque GSB, Macedo C, Aras R; Análise estatística e Redação do manuscrito: Freitas EL, Sampaio ES, Oliveira MMC, Aras R; : Freitas EL, Sampaio ES, Oliveira MMC, Aras R; Revisão crítica do manuscrito quanto ao conteúdo intelectual importante: Aras R.

\section{Potencial Conflito de Interesses}

Declaro não haver conflito de interesses pertinentes.

\section{Fontes de Financiamento}

O presente estudo não teve fontes de financiamento externas.

\section{Vinculação Acadêmica}

Este artigo é parte de dissertação de Mestrado de Emanoela Lima Freitas pela Universidade Federal da Bahia. 


\section{Referências}

1. Romero JR, Wolf PA. Epidemiology of stroke: legacy of the Framingham Heart Study. Glob Heart. 2013;8(1):67-75.

2. Glotzer TV, Hellkamp AS, Zimmerman J, Sweeney MO, Yee R, Marinchak $\mathrm{R}$, et al. Atrial high rate episodes detected by pacemaker diagnostics predict death and stroke: report of the Atrial Diagnostics Ancillary Study of the MOde Selection Trial (MOST). Circulation. 2003;107(12):1614-19.

3. Glotzer TV, Daoud EG, Wyse DG, Singer DE, Ezekowitz MD, Hilker C, et al. The relationship between daily atrial tachyarrhythmia burden from implantable device diagnostics and stroke risk: the TRENDS study. Circ Arrhythm Electrophysiol. 2009;2(5):474-80.

4. Healey JS, Connolly SJ, Gold MR, Israel CW, Gelder ICV, Capucci A, et al. ASSERT Investigators. Subclinical atrial fibrillation and the risk of stroke. N Engl J Med. 2012;366(2):120-9.

5. Camm AJ, Simantirakis E, Goette A, Lip GYH, Vardas P, Calvert M, et al. Atrial high-rate episodes and stroke prevention. Europace. 2017;19(2):169-79.

6. Surapaneni P, Safadi A, Contractor T, Patel MB, Thakur RK. Device-detected atrial fibrillation-Perils and Pitfalls: an update. Cardiol Clin. 2016;34(2):299-306.

7. Freedman B, Boriani G, Glotzer TV, Healey J, Kirchhof P, Potpara TS. Management of atrial high-rate episodes detected by cardiac implanted electronic devices. Nat Rev Cardiol. 2017;14(12):701-14

8. Capucci A, Santini M, Padeletti L, Gulizia M, Botto G, Boriani G, et al. Monitored atrial fibrillation duration predicts arterial embolic events in patients suffering from bradycardia and atrial fibrillation implanted with antitachycardia pacemakers. J Am Coll Cardiol. 2005;46(10):1913-20.

9. Ziegler PD, Glotzer TV, Daoud EG, Wyse DG, Singer DE, Ezekowitz MD, et al. Incidence of newly detected atrial arrhythmias via implantable devices in patients with a history of thromboembolic events. Stroke. 2010;41(2):256-60.

10. Ziegler PD, Glotzer TV, Daoud EG, Singer DE, Ezekowitz MD, Hoyt RH, et al. Detection of previously undiagnosed atrial fibrillation in patients with stroke risk factors and usefulness of continuous monitoring in primary stroke prevention. Am J Cardiol. 2012;110(9):1309-14.

11. World Health Organization. Chagas disease (American trypanosomiasis) [Internet]. Geneva: WHO; 2015 [acesso em 12 Agosto 2017]. Disponível em: http://www.who.int/mediacentre/factsheets/fs340/en/.

12. Martins-Melo FR, Ramos Junior AN, Alencar CH, Heukelbach J. Multiple causes of death related to Chagas' disease in Brazil, 1999 to 2007. Rev Soc Bras Med Trop. 2012;45(5):591-6.

13. Garzon SAC, Lorga, AM, Nicolau JC. Electrocardiography in Chagas' heart disease. Sao Paulo Med. J. 1995;113(2):802-13.

14. Dias JCP, Ramos Jr AN, Gontijo ED, Luqueti A, Shikanai-Yasuda MA, Coura JR, et al. Brazilian Consensus on Chagas Disease, 2015. Epidemiol Serv Saúde. 25(n esp):7-86.

15. Sousa AS, Xavier SS, Freitas GR, Hasslocher-Moreno A.. Prevention strategies of cardioembolic ischemic stroke in Chagas' disease. Arq Bras Cardiol. 2008;91(5):306-10
16. Lip GYH, Nieuwlaat R, Pisters R, Lane DA, Crijns HJGM. Refining clinical risk stratification for predicting stroke and thromboembolism in atrial fibrillation using a novel risk fator-based approach: The Euro Heart Survey on Atrial Fbrillation. Chest. 2010;137(2):263-72.

17. Barold SS, Levine PA. Pacemaker repetitive nonreentrant ventriculoatrial synchronous rhythm. A review. J Interv Card Electrophysiol. 2001;5(1):45-58

18. Kohno R, Abe H, Oginosawa Y, Tamura M, Takeuchi M, Nagamoto T, et al. Reliability and characteristics of atrial tachyarrhythmias detection in dual chamber pacemakers. Circ J. 2011;75(5):1090-7.

19. Kaufman ES, Israel CW, Nair GM, Armaganijan L, Divakaramenon S Mairesse $\mathrm{GH}$, et al. Positive predictive value of device-detected atrial highrate episodes at different rates and durations: an analysis from ASSERT. Heart Rhythm. 2012;9(8):1241-6.

20. Benezet-Mazuecos J, Rubio JM, Cortés M, Iglesias JA, Calle S, Vieja JJ, et al. Silent ischaemic brain lesions related to atrial high rate episodes in patients with cardiac implantable electronic devices. Europace. 2015;17(3):364-9.

21. Benezet-Mazuecos J, Iglesias JA, Cortes M, Vieja JJ, Rubio JM, SanchesBorque $\mathrm{P}$, et al. Silent brain infarcts in high blood pressure patients with cardiac implantable electronic devices: unmasking silent atrial fibrillation. J Hypertens. 2016;34(2):338-44.

22. Marfella R, Sasso FC, Siniscalchi M, Cirillo M, Paolisso P, Sardu C, et al. Brie episodes of silent atrial fibrillation predict clinical vascular brain disease in type 2 diabetic patients. J Am Coll Cardiol. 2013;62(6):525-30.

23. Kannel WB, Abbott RD, Savage DD, McNamara PM. Epidemiologic features of chronic atrial fibrillation: the Framingham study. N Engl J Med. 1982;306(17):1018-22.

24. Marcolino MS, Palhares DM, Benjamin EJ, Ribeiro AL. Atrial fibrillation: prevalence in a large database of primary care patients in Brazil. Europace. $2015 ; 17(12): 1787-90$.

25. Vermeer SE, Longstreth WT Jr, Koudstaal PJ. Silent brain infarcts: a systematic review. Lancet Neurol. 2007:6(7):611-9.

26. Vanassche T, Lauw MN, Eikelboom JW, Healey JS, Hart RG, Alings M, et al Risk of ischaemic stroke according to pattern of atrial fibrillation: analysis of 6563 aspirin-treated patients in ACTIVE-A and AVERROES. Eur Heart J. 2015;36(5):281-8.

27. Shanmugam N, Boerdlein A, Proff J, Ong P, Valencia O, Maier SKG, et al. Detection of atrial high-rate events by continuous home monitoring:clinical significance in the heart failure-cardiac resynchronization therapy population. Europace. 2012;14(2):230-7.

28. Boriani G, Glotzer TV, Santini M, West TM, Melis MD, Sepsi M, et al Device-detected atrial fibrillation and risk for stroke: an analysis of $>10,000$ patients from the SOS AF project (Stroke preventiOn Strategies based on Atrial Fibrillation information from implanted devices). Eur Heart J. 2014;35(8):508-16. 